EPJ Web of Conferences 13, 06002 (2011)

DOI: $10.1051 /$ epjconf/20111306002

(C) Owned by the authors, published by EDP Sciences, 2011

\title{
Multi-particle interactions within the UrQMD approach
}

\author{
G. Burau ${ }^{1,2, a}$, G. Gräf ${ }^{1,2}$, H. Petersen ${ }^{2,3}$, and M. Bleicher ${ }^{1,2}$ \\ 1 Frankfurt Institute for Advanced Studies, Ruth-Moufang-Str. 1, 60438 Frankfurt am Main, Germany \\ 2 Institut für Theoretische Physik, Goethe-Universität, Max-von-Laue-Str. 1, 60438 Frankfurt am Main, Germany \\ 3 Present address: Department of Physics, Duke University, Durham, North Carolina 27708-0305, United States
}

\begin{abstract}
A mechanism for locally density-dependent dynamic parton rearrangement and fusion has been implemented into the Ultrarelativistic Quantum Molecular Dynamics (UrQMD) approach. The same mechanism has been previously built in the Quark Gluon String Model (QGSM). This rearrangement and fusion approach based on parton coalescence ideas enables the description of multi-particle interactions, namely $3 \rightarrow 3$ and $3 \rightarrow$ 2 , between (pre)hadronic states in addition to standard binary interactions. The UrQMD model (v2.3) extended by these additional processes allows to investigate implications of multi-particle interactions on the reaction dynamics of ultrarelativistic heavy ion collisions. The mechanism, its implementation and first results of this investigation are presented and discussed.
\end{abstract}

\section{Introduction}

The study of nucleus-nucleus collisions at high energies is a prosperous and exciting field of experimental and theoretical research since many years because those collisions provide a promising way to produce matter with high densities and high temperatures. In order to explore this excited "nuclear" matter and to reveal its properties, the experimental research is reliant on theoretical investigations and vice versa, see for example [1,2] and references therein. Currently heavy ion reactions at very high initial collision energies are in the focus of these investigations, namely collisions of gold ions with center of mass energies of up to $E_{\mathrm{cm}}=200 \mathrm{GeV}$ at the Relativistic Heavy Ion Collider (RHIC) and, very recently started, collisions of lead nuclei with even higher energies of few $\mathrm{TeV}$ per nucleon at the Large Hadron Collider (LHC). Here the regime of very high temperatures $T$ but low baryon chemical potential $\mu_{\mathrm{B}}$ is explored. On the other side, the low energy (high $\mu_{\mathrm{B}}$ ) program at CERN-SPS has shown a culmination of exciting results in the area of lower $T$ but high $\mu_{\mathrm{B}}$. Therefore further detailed studies at CERN-SPS, BNL-RHIC and the Facility for Antiproton and Ion Research (FAIR) near the GSI Helmholtz Center for Heavy Ion Research will be dedicated to this energy regime.

In this context it is important to obtain an understanding of the reaction dynamics in terms of microscopic approaches. One class of these approaches that aims at the description of heavy ion reactions consistently from the initial until the final state employs relativistic transport theory [3-10]. This microscopic description has been applied quite successfully to the partonic as well as hadronic stage of the reaction. However, most transport approaches are restricted to binary, $2 \rightarrow n$, scattering processes. So it be-

\footnotetext{
a e-mail: burau@th.physik.uni-frankfurt.de
}

comes questionable whether this restriction to two-particle interactions is still justified, if the density of particles produced, especially in the early stage of nucleus-nucleus collisions, increases. A few attempts to include multi-particle interactions into microscopic approaches have been already proposed [7,11-17]. Nevertheless, this extension of transport theory is still debated.

In this work an extension of the UrQMD approach by three-particle interactions, $3 \rightarrow n(n \leq 3)$, realized via dynamic parton rearrangement processes is presented. This extension is based on a mechanism which has been previously implemented in a similar microscopic transport approach, namely the Quark Gluon String Model (QGSM) [16-18]. Thus the paper is organized as follows: In Sect. 2 the current state-of-the-art UrQMD approach (version 2.3) is briefly reviewed. In Sect. 3 the main concept of the parton rearrangement processes and their implementation into UrQMD are summarized. First results from the extended model focussing on key characteristics of the implemented multi-particle interactions are discussed in Sect. 4. Finally, a summary and conclusions are given in Sect. 5 .

\section{UrQMD in a nutshell}

UrQMD, the Ultrarelativistic Quantum Molecular Dynamics approach $[3,5,19]$, is a non-equilibrium hadronic transport approach using Monte-Carlo simulation techniques in order to describe consistently heavy ion reactions from the beginning (initialization of projectile and target nuclei) until the end (final state of the system). The approach simulates multiple interactions of ingoing and newly produced particles and constitutes an effective solution of the relativistic Boltzmann equation. The underlying degrees of freedom are strings and hadrons that are excited in high energetic binary collisions.

This is an Open Access article distributed under the terms of the Creative Commons Attribution-Noncommercial License 3.0, which permits unrestricted use, distribution, and reproduction in any noncommercial medium, provided the original work is properly cited. 
Projectile and target nuclei are initialised according to a Woods-Saxon profile in coordinate space. Fermi momenta for each nucleon are randomly assigned in the rest frame of the projectile and target nucleus, respectively. The initial nucleons and, later on, newly produced hadrons are propagated on straight lines until the collision criterion is fulfilled:

$$
d_{\text {trans }} \leq d_{0}=\sqrt{\sigma_{\text {tot }} / \pi}, \quad \sigma_{\text {tot }}=\sigma(\sqrt{s}, \text { type })
$$

If the covariant relative distance $d_{\text {trans }}$ between two particles is smaller than a critical distance, given by the corresponding total cross section, a collision of those two particles takes place. The elementary cross sections are fitted according to available data, parametrized using the additive quark model, or calculated by detailed balance.

Particle production is described by excitation and fragmentation of classical color strings with (anti)quarks or (anti)diquarks at their ends and by excitation and decay of hadronic resonances. Excitation and fragmentation of the strings are treated according to the Lund model. Hard collisions with large momentum transfer, i.e. larger $1.5 \mathrm{GeV}$, are treated using PYTHIA. A phase transition to a state of free quarks and gluons is not explicitly incorporated into the approach. Within UrQMD all hadrons and resonances up to $2.2 \mathrm{GeV}$ are considered. This microscopic transport approach generates the full space-time dynamics of the strings and hadrons; the full phase space information is available at every stage of the simulated heavy ion collision. After all, it successfully describes different observables in a broad range of collision systems and energies [20]. A much more detailed description of the current standard version of UrQMD (v2.3), which served as basis for the extension and study presented in this work, together with a compilation of its results compared to experimental data can be found in [19].

Like other (hadronic) transport approaches of this kind, only binary collisions, $2 \rightarrow n$ processes, and one-particle decays are considered to keep the calculation numerically tractable. However, this restriction to binary collisions assumes a system with large mean free paths of the particles. As mentioned in Sect. 1, this assumption might not be justified in the early stages of heavy ion reactions anymore. In this regime the particle densities can be very high. Observables that are sensitive to the properties of the early stage of the reaction or to the thermal and chemical equilibration during the reaction - like elliptic flow, HBT radii and strangeness, in particular the number of produced multistrange baryons - hint to the fact that transport models considering only binary interactions may not be sufficient to describe the entire dynamics of heavy ion collisions at high energies [21-25]. Thus the consideration of additional interactions among three particles, realized, e.g., by rearrangement processes, might fit better to characterize the hot and very dense system. The specific implementation of these processes, developed previously [16-18] within a transport approach similar to UrQMD, will be summarized in the following section.

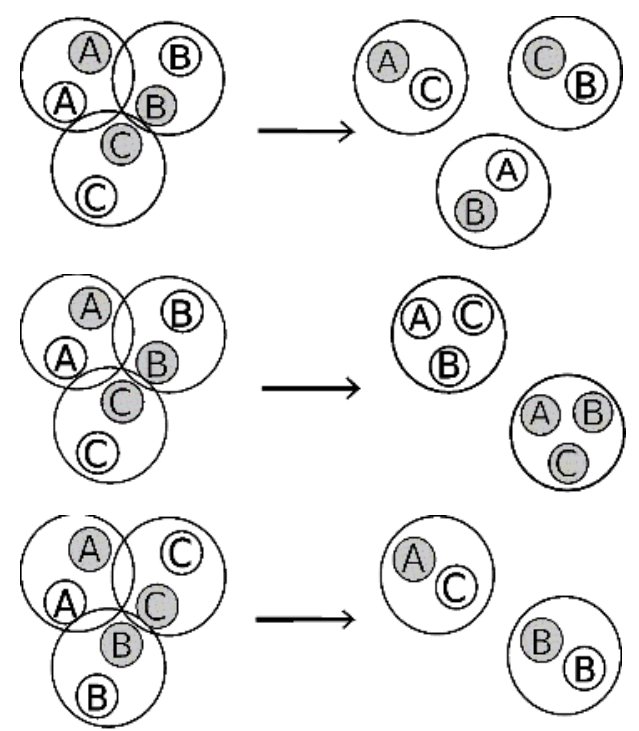

Fig. 1. Schematic representation of the implemented rearrangement processes: recombination of partons (upper panel), meson fusion to (anti)baryons (middle), and quark-antiquark annihilation processes (lower panel) [26]. A more detailed explanation is given in the text.

\section{Dynamic parton rearrangement}

Since the UrQMD approach describes particle production by excitation and fragmentation of strings with different (anti)quarks or (anti)diquarks, in the following also referred to as partons, it provides like the QGSM approach a framework for the implementation of locally density-dependent parton rearrangement processes. These three-particle interactions should occur in the high density stages of heavy ion reactions additionally to the standard binary collisions. Hence the basic idea is the following: Above a critical local particle density, three hadrons - either formed or unformed according to their treatment in UrQMD - satisfying corresponding constrains are decomposed into their constituents, i.e. their partons: quark and antiquark for mesons, (anti)quark and (anti)diquark for baryons and antibaryons, respectively. These partons of those hadrons are then allowed to rearrange themselves into new hadronic states which is schematically shown in Fig. 1. All particles are allowed to interact via rearrangement, even non-formed hadrons (prehadrons). To trigger these processes, the critical local particle density is effectively taken into account due to a given critical distance between the particles: If the spatial distance of three (pre)hadrons in their center of mass frame is less than $0.85 \mathrm{fm}$ this three (pre)hadrons are generally allowed to be involved in a rearrangement process. According to usual Monte Carlo methods one triplet of all possible (pre)hadron triplets, which satisfy this spatial constrain, is randomly chosen for rearrangement. Each parton of the decomposed (pre)hadrons gets a momentum fraction $z$ of the initial longitudinal momentum of its (pre) hadron and a transverse momentum $p_{T}$. Both, $z$ and $p_{T}$ are generated from the standard parton distribution functions used within UrQMD. Additionally to the spatial constrain, rearrangement processes require also an overlap of the par- 


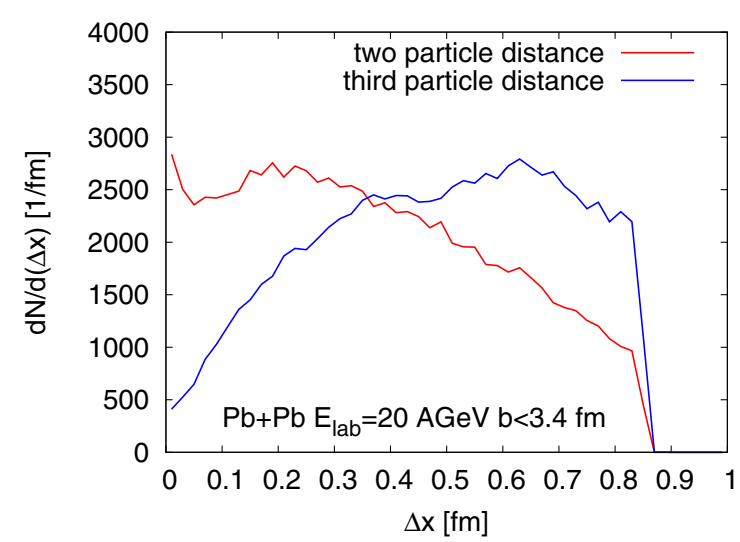

Fig. 2. Distribution of distances between two particles, i.e. (pre)hadrons, and distribution of distances to a third particle involved in rearrangement processes (exemplified for central $\mathrm{Pb}+\mathrm{Pb}$ collisions at $\left.E_{\text {lab }}=20 A \mathrm{GeV}\right)$.

ticipating partons in the momentum space. The distance in momentum space between rearrangeing partons is evaluated in the center of mass frame, too. The allowed difference in momentum is given by the uncertainty principle, $\Delta x \Delta p \geq 1$ where the spatial distance between the participating (pre)hadrons, shown in Fig. 2 is used. Furthermore, rearrangement processes can take place only if the quantum numbers allow the partons to rearrange into new (pre) hadronic states. The implemented mechanism always conserves the quantum numbers, in particular the electric charge and the baryon number, and ensures that only physical particles can be final states, e.g. no final diquark states are possible. This approach does not create a system of "free partons". If the outcome of a rearrangement process would yield any unphysical particle it cannot take place. Either an other valid rearrangement process is chosen or, if none is possible, no rearrangement occurs. If no rearrangement processes take place standard elastic or inelastic scatterings, i.e. binary collisions, occur.

In general three processes are possible as depicted in Fig. 1: (i) Parton recombination in terms of "reshuffling" of the involved partons forms new (pre)hadrons. The number of mesons and (anti)baryons in the initial and final states is hereby conserved, i.e. three hadrons go in and three go out. So this is a $3 \rightarrow 3$ process. (ii) Meson fusion forms a pair of baryon and antibaryon by combining the quarks of three decomposed mesons to a baryon and the remaining three antiquarks to an antibaryon. This is a process which reduces the number of final hadrons because three particles (mesons) are in the initial state but only two particles are in the final state, i.e. $3 \rightarrow 2$. (iii) Additionally, a quark-antiquark pair of same flavor may annihilate during the rearrangement process with a given probability. In this case the energy and momentum of the annihilated quarkantiquark pair is redistributed to the new mesons formed by the remaining quarks and antiquarks. This process effectively implements another $3 \rightarrow 2$ reaction. Details on the probability for these annihilation processes are discussed in $[16,17]$.

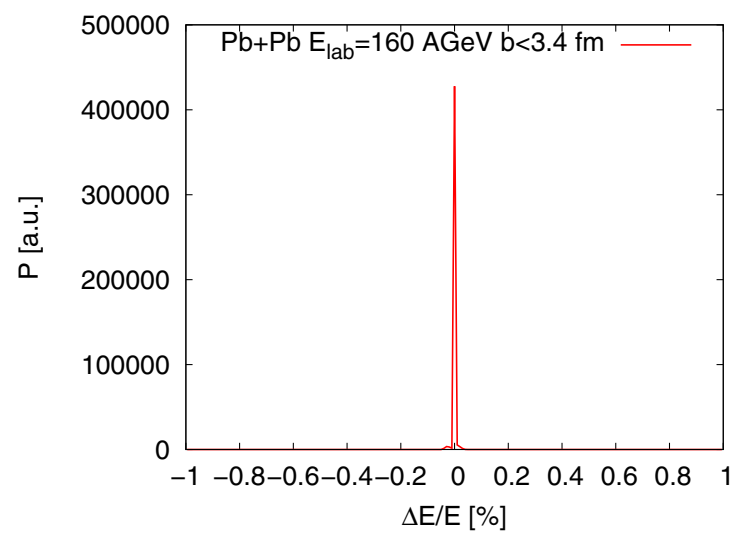

Fig. 3. Distribution of the relative deviation of the energy, $\Delta E / E$ (in percent), in rearrangement processes (exemplified for central $\mathrm{Pb}+\mathrm{Pb}$ collisions at $E_{\text {lab }}=160 \mathrm{AGeV}$ ). The absolute deviation $\triangle E$ is the difference between incoming and outgoing particles.

Charge conservation is automatically guaranteed within this approach. Conserving energy and three-momentum simultaneously is not possible for the rearrangement processes described above. However, applying a rescaling procedure for the momenta in the center of mass system of the produced hadronic states, simultaneous conservation of energy and three-momentum can be ensured with very high precision. Fig. 3 shows the result for the relative deviation of the energy. In order to conserve the initial energy the three-momentum components of all particles involved in a particular reaction are scaled by a constant factor which is determined iteratively, see $[18,26]$. Moreover, by such a procedure ratios of momenta like anisotropic flow coefficients remain unchanged, i.e. anisotropic flow is not artificially influenced by the momentum rescaling.

All rearrangement processes, i.e. interactions among three particles, might very frequently happen as long as the local particle density of the medium is high enough. Accordingly, these rearrangement processes become less important with ongoing expansion of the fireball when the system increasingly thins out. In this spirit the UrQMD approach extended by the locally density-dependent parton rearrangement processes considers - within its limitations interactions of three particle in addition to binary collisions and allows to investigate possible implications on the reaction dynamics of heavy ion collisions at high energies from a microscopical point of view including comparisons with results from previous studies within the upgraded QGSM [16-18].

\section{Checks and first results}

The work presented here focused on the implementation of multi-particle interactions via the rearrangement processes and its testing with respect to key characteristics of the extended approach. For this study, $\mathrm{Au}+\mathrm{Au}$ and $\mathrm{Pb}+\mathrm{Pb}$ collisions for a broad range of collision energies and impact parameters $b<3.4 \mathrm{fm}$ have been simulated [26]. 


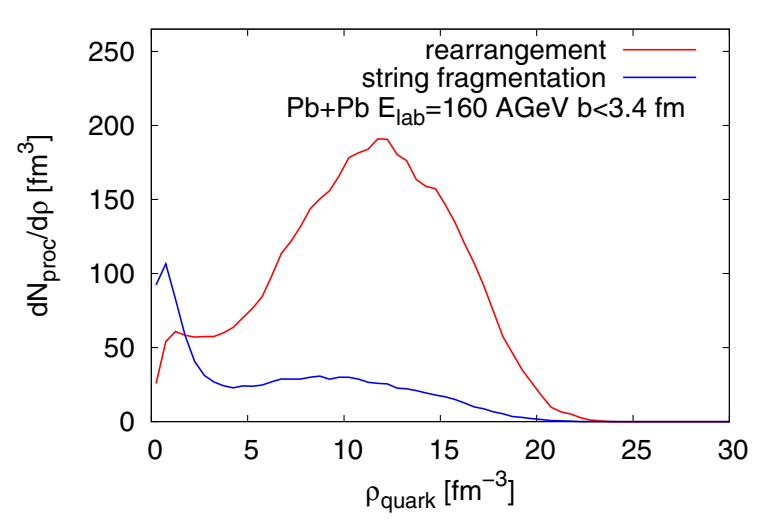

Fig. 4. Distribution of rearrangement processes and string fragmentation processes, respectively, as function of the quark density (exemplified for central $\mathrm{Pb}+\mathrm{Pb}$ collisions at $E_{\text {lab }}=$ $160 \mathrm{AGeV})$.

Since the implemented rearrangement processes should mostly happen in the high density stage of heavy ion collisions, the density dependence of these processes has been checked first. The corresponding result, i.e. the distribution of the number of rearrangement processes with respect to the local quark density $\rho_{\text {quark }}=\rho_{q+\bar{q}}{ }^{1}$, is exemplarily shown in Fig. 4 for central $\mathrm{Pb}+\mathrm{Pb}$ collisions at an energy of $E_{\mathrm{lab}}=160 \mathrm{AGeV}$ in the laboratory frame. This distribution clearly shows a broad peak at higher quark densities while the analogous distribution for the standard string fragmentation processes from inelastic binary collisions is much flatter and smaller in this region, but the latter dominates in the small density regime. So, rearrangement happens indeed mostly in regions of high particle densities.

In order to get deeper insight into the dynamics of the simulated collisions, the temporal evolution of the implemented interaction processes has been studied next. Fig. 5 depicts the time dependence of the number of all threeparticle interactions (rearrangement) and the number of all other standard processes considered in UrQMD-2.3, respectively. Results for central $\mathrm{Au}+\mathrm{Au}$ and $\mathrm{Pb}+\mathrm{Pb}$ collisions at four different energies, namely $E_{\mathrm{cm}}=130 \mathrm{AGeV}$ and $E_{\text {lab }}=160 \mathrm{AGeV}, 20 \mathrm{AGeV}, 4 \mathrm{AGeV}$, are shown top down. The highest interaction rates of both, i.e. one-/twoand three-particle interactions, occur at nearly same time during the evolution of the heavy ion reaction. This finding holds for all collision energies. The main difference is the significance of the processes at different energies. In heavy ion collisions at low energies, the amount of rearrangement processes is practically negligible, see panel at the bottom of Fig. 5. Binary particle collisions and resonance decays clearly dominate in this regime. But with increasing energy of the colliding ions rearrangement among three particles gains more and more importance, which reflects the increase in particle density in the early stage of those collisions. Finally, rearrangement processes dominate the interactions at energies above $E_{\mathrm{lab}} \approx 20 \mathrm{AGeV}$, especially

\footnotetext{
${ }^{1}$ In the appendix of [26] it has been explained how this quark density is determined.
}
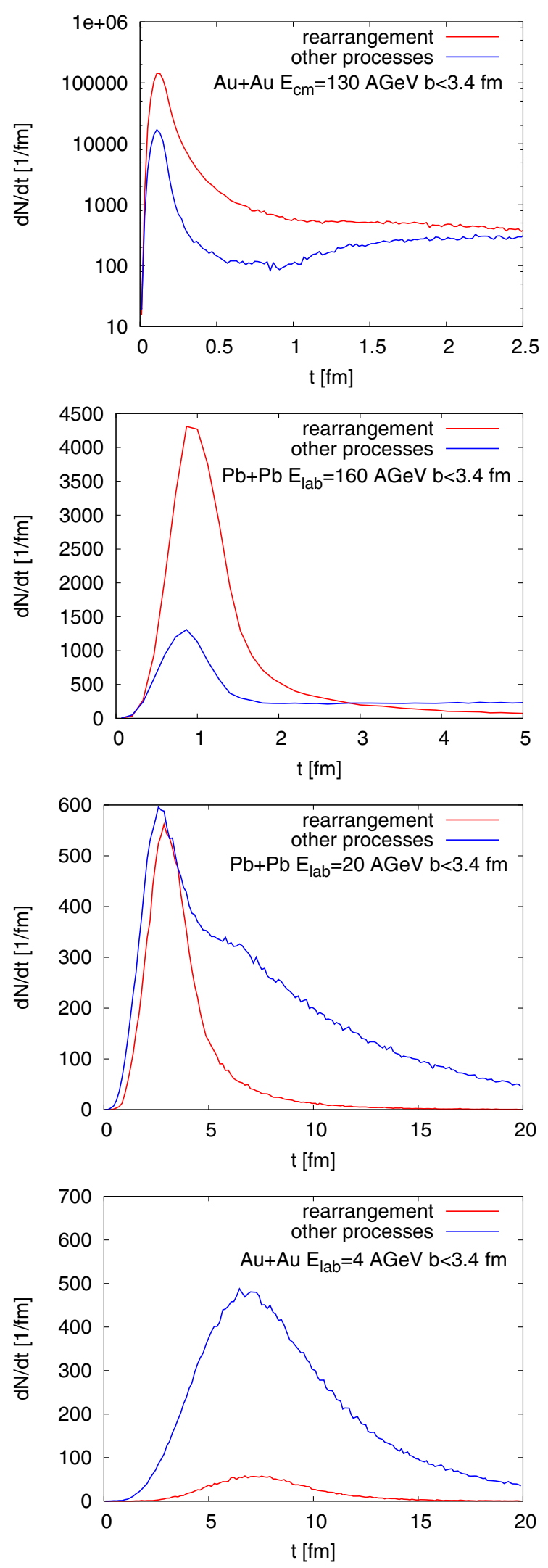

Fig. 5. Time dependence of rearrangement processes (all threeparticle interactions) compared to all other processes (twoparticle interactions and resonance decays implemented in UrQMD-2.3) in central $\mathrm{Au}+\mathrm{Au}$ and $\mathrm{Pb}+\mathrm{Pb}$ collisions, respectively. Results for four different collision energies, decreasing top down, are shown. 


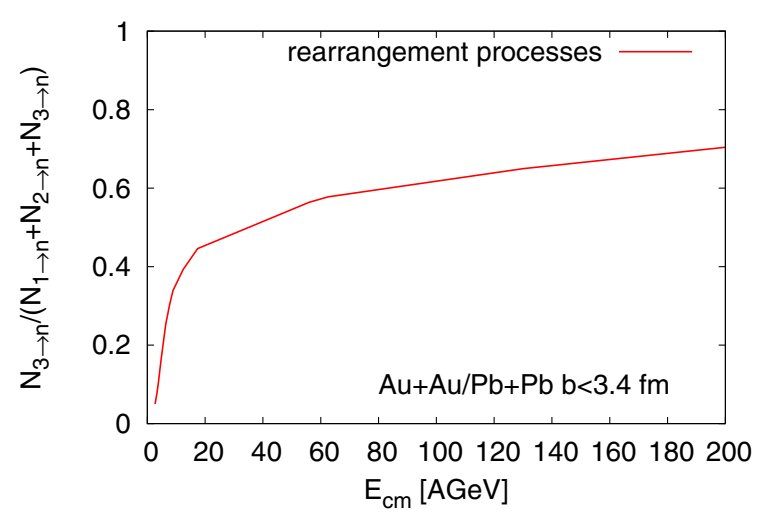

Fig. 6. Number of three-particle interactions, i.e. rearrangement processes, relative to the number of all implemented interactions and its dependence on the center of mass energy for central $\mathrm{Au}+\mathrm{Au}$ and $\mathrm{Pb}+\mathrm{Pb}$ collisions.

during the first few $\mathrm{fm} / \mathrm{c}$ of the heavy ion reaction (upper panels of Fig. 5) where very high densities are expected.

The finding shown in Fig. 6 also reflects the increasing influence of multi-particle interactions at higher energies. This figure displays the energy dependence of the relative number of rearrangement processes, $N_{3 \rightarrow n}$, with respect to the number of all implemented interactions, $N_{1 \rightarrow n}+N_{2 \rightarrow n}+$ $N_{3 \rightarrow n}$, extracted from simulated $\mathrm{Au}+\mathrm{Au}$ and $\mathrm{Pb}+\mathrm{Pb}$ collisions at various center of mass energies. Starting from a few percent level at very low energy, the depicted ratio drastically grows up to about $40 \%$ at top SPS energy, i.e. $E_{\text {lab }}=20 \mathrm{AGeV}$. Then it rises even further with increasing energy, but this rise shows a moderated slope. Hence distinct implications on the reaction dynamics and on relevant observables are expected for heavy ion reactions at energies higher than SPS energy.

Last but not least the contributions of the three kinds of rearrangement processes introduced in Sect. 3, including recombination, quark-antiquark annihilation, and meson fusion, have been investigated. The interesting point here is that the different sub-processes could influence the dynamics of the system in their specific way. The numbers of the accordant sub-process divided by the number of all rearrangement processes $\left(N_{3 \rightarrow n}\right)$ are calculated for the same $\mathrm{Au}+\mathrm{Au}$ and $\mathrm{Pb}+\mathrm{Pb}$ collisions mentioned above. The corresponding results are presented in Fig. 7. Recombination, i.e. reshuffling of partons among three (pre)hadrons, is the dominant three-particle process at all collision energies, see upper panel of Fig. 7, which increases significantly the interaction rates during the early high density stage of the heavy ion reaction. Therefore this process is expected to enhance the pressure in the early stage and to drive the system to a faster kinetic equilibrium compared to calculations with the standard UrQMD-2.3 approach. The number of processes considering quark-antiquark annihilation (panel in the middle of Fig. 7) are already two orders of magnitude smaller with respect to recombination. This sub-process should lead to some accretion of transverse momenta of the involved particles since the energy released by annihilation is redistributed among the remain-
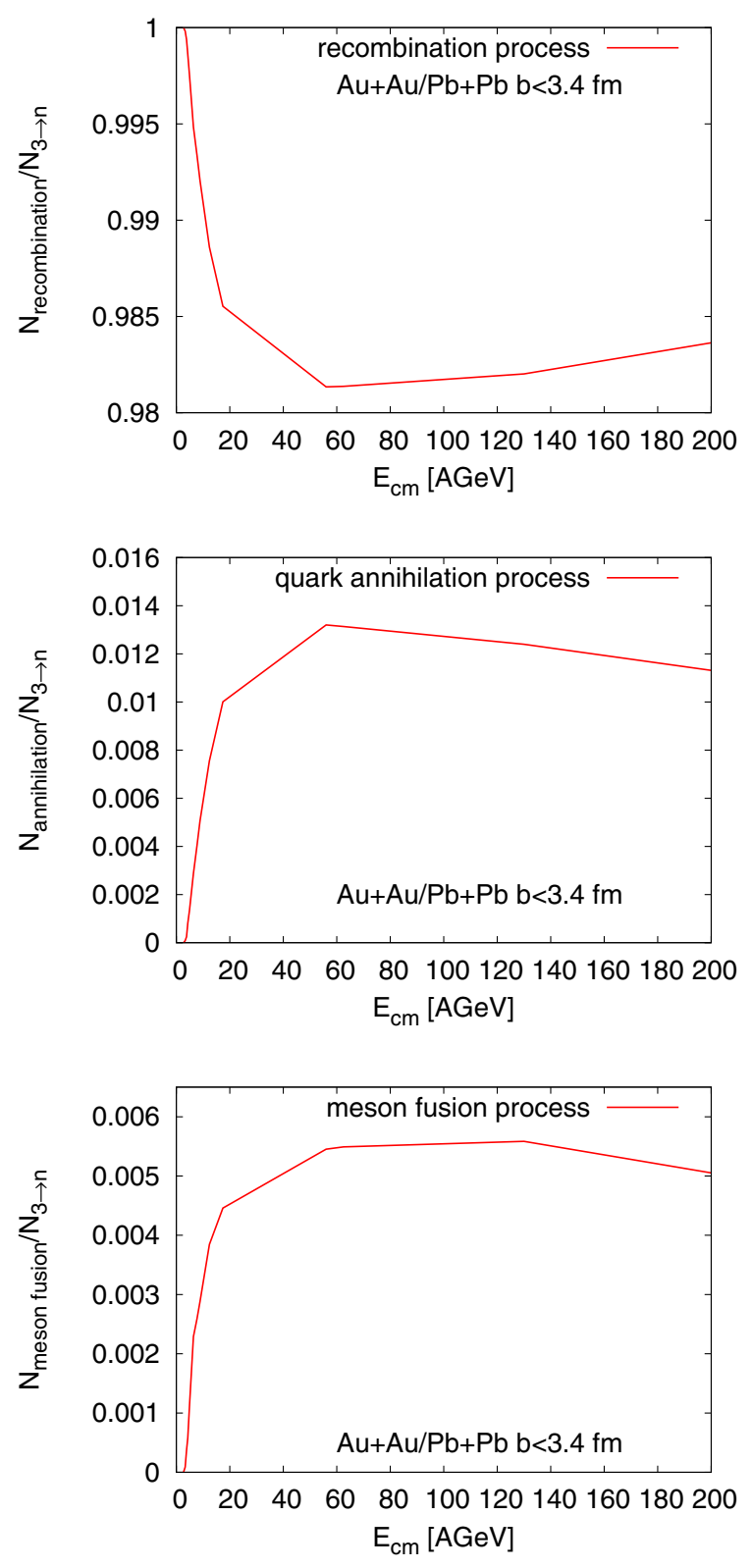

Fig. 7. Relative number of recombination processes (upper panel), quark-antiquark annihilation (middle), and meson fusion processes (lower panel) with respect to all three-particle interactions (rearrangement) and their dependence on the center of mass energy (central $\mathrm{Au}+\mathrm{Au}$ and $\mathrm{Pb}+\mathrm{Pb}$ collisions).

ing two (pre)hadrons - either directly as kinetic energy or indirectly via the formation and decay of resonance states. Together with recombination this might increase the elliptic flow $v_{2}$ of the final hadrons which has been found to be intimately related to the dynamics of the system. Previous studies within a QGSM transport approach extended by rearrangement processes support these expectations [16-18]. Meson fusion processes are finally suppressed by an additional factor of ten as shown in the lower panel of Fig. 7. However, meson fusion is still important although it is only a tiny fraction of the implemented three-particle interac- 
tions. As explained in Sect. 3, the fusion of three mesons produces a pair of a baryon and an antibaryon. Especially antibaryons are rarely produced by the standard binary processes so that even small changes can become important in this sector. For example, it is expected that this mechanism influences amongst others the (anti)hyperon production. First preliminary findings for heavy ion collisions simulated with the modified UrQMD approach hint towards an enhancement of those hadronic states due to meson fusion [26]. But this question together with a detailed investigation of the consequences for observables which can be compared to experimental data deserves further studies.

\section{Summary and conclusions}

An extension of the UrQMD approach by implementing three-particle interactions, $3 \rightarrow n(n \leq 3)$, realized via dynamic parton rearrangement has been presented in this work. All particles, namely formed and unformed hadrons (latter often referred to as prehadrons) are allowed to participate in these rearrangement processes while in the standard binary collisions only formed hadronic states are involved. All quantum numbers such as electric charge and baryon number are conserved within this approach. Simultaneous conservation of energy and three-momentum is ensured by momentum rescaling.

A first proof-of-principle study employing this extended transport approach focussed on key characteristics of the implemented multi-particle interactions. Therefore central collisions of lead and gold nuclei, respectively, have been simulated for a broad range of energies. It has been demonstrated that rearrangement occurs mainly in the high density region of nucleus-nucleus reactions and dominates the interactions during the first few $\mathrm{fm} / \mathrm{c}$ of the reactions at collision energies above $E_{\mathrm{lab}} \approx 20 \mathrm{AGeV}$. Finally, recombination such as $3 M \rightarrow 3 \tilde{M}$ have been found to be by far the dominant sub-process compared to quark-antiquark annihilation processes, $3 M \rightarrow 2 \tilde{M}$, and meson fusion, $3 M \rightarrow$ $B \bar{B}^{2}$. Altogether, all three sub-processes are expected to verifiably influence the reaction dynamics and related observables as preliminary findings indicate. More detailed investigations within the UrQMD approach extended by multi-particle interactions are needed to provide more insight into implications on physical observables like, e.g., particle multiplicities, spectra, and anisotropic flow parameters.

\section{Acknowledgement}

The authors are grateful to S.A. Bass and J. Bleibel for fruitful discussions. This work was supported by the GSI, BMBF, and by the Hessian LOEWE initiative through the Helmholtz International Center for FAIR (HIC for FAIR), the Helmholtz Graduate School for Heavy Ion Research (HGS-HIRe for FAIR), and the Helmholtz Research School

\footnotetext{
${ }^{2} M, \tilde{M}, B$, and $\bar{B}$ denote mesons, baryons and antibaryons,
} respectively. on Quark Matter Studies (H-QM). The Center for Scientific Computing (CSC) at Goethe-Universität Frankfurt is acknowledged for providing the computational resources.

\section{References}

1. P. Stankus, D. Silvermyr, S. Sorensen, and V. Greene, (editors), Proceedings of the 21st International Conference on Ultrarelativistic Nucleus-Nucleus Collisions - Quark Matter 2009, (Knoxville, USA, 2009) [Nucl. Phys. A 830, 1c-968c (2009)]

2. B. Friman, C. Höhne, J. Knoll, S. Leupold, J. Randrup, R. Rapp, and P. Senger (editors), The CBM Physics Book - Compressed Baryonic Matter in Laboratory Experiments, (Springer, Heidelberg, 2011) [Series: Lecture Notes in Physics Vol. 814, 1-960 (2011)]

3. S. A. Bass et al., Prog. Part. Nucl. Phys. 41, (1998) 255 [Prog. Part. Nucl. Phys. 41, 225 (1998)]

4. J. Geiss, W. Cassing, and C. Greiner, Nucl. Phys. A 644, 107 (1998)

5. M. Bleicher et al., J. Phys. G 25, 1859 (1999)

6. D. Molnar and P. Huovinen, Phys. Rev. Lett. 94, 012302 (2005)

7. Z. Xu and C. Greiner, Phys. Rev. C 71, 064901 (2005)

8. Z.W. Lin et al., Phys. Rev. C 72, 064901 (2005)

9. G. Burau et al., Phys. Rev. C 71, 054905 (2005)

10. J. Bleibel, G. Burau, A. Fässler, and C. Fuchs, Nucl. Phys. A 767, 218 (2006)

11. P. Danielewicz, Phys. Rev. C 42, 1564 (1990)

12. H.W. Barz, B. Kämpfer, Nuc. Phys. A 683, 594 (2001)

13. C. Greiner, S. Leupold, J. Phys. G 27, L95 (2001)

14. W. Cassing, Nuc. Phys. A 700, 618 (2002)

15. A.B. Larionov, O. Buss, K. Gallmeister, and U. Mosel, Phys. Rev. C 76, 044909 (2007)

16. J. Bleibel, G. Burau, A. Fässler, C. Fuchs, Phys. Rev. C 76, 024912 (2007)

17. J. Bleibel, G. Burau, C. Fuchs, Phys. Lett. B 659, 520 (2008)

18. J. Bleibel, Untersuchung stark wechselwirkender Materie mittels ultrarelativistischer Schwerionenstöße (Dissertation, Eberhard-Karls-Universität, Tübingen, 2007)

19. H. Petersen et al., arXiv:0805.0567 [hep-ph]

20. E. L. Bratkovskaya et al., Phys. Rev. C 69, 054907 (2004)

21. M. Bleicher and H. Stöcker, Phys. Lett. B 526, 309 (2002)

22. U. W. Heinz and P. F. Kolb, arXiv:hep-ph/0204061

23. D. Molnar and M. Gyulassy, Phys. Rev. Lett. 92, 052301 (2004)

24. H. Petersen, Q. Li, X. Zhu and M. Bleicher, Phys. Rev. C 74, 064908 (2006)

25. Q. Li, M. Bleicher and H. Stöcker, Phys. Lett. B 659, 525 (2008)

26. G. Gräf, Multi-Teilchen-Wechselwirkungen in Schwerionenkollisionen (Diplomarbeit, Goethe-Universität, Frankfurt am Main, 2009) 